TAIWANESE JOURNAL OF MATHEMATICS

Vol. 16, No. 1, pp. 47-70, February 2012

This paper is available online at http://tjm.math.ntu.edu.tw

\title{
THE INITIAL VALUE PROBLEM AROSE FROM UNPERTURBED HUMAN TUMOUR CELL LINES
}

\author{
Yu-Hsien Chang, Kang Fang and Guo-Chin Jau
}

\begin{abstract}
To learn more of the phase distributions in unperturbed human tumour cells is a prerequisite prior to understanding of those in the perturbed cells. The work is important in understanding the efficiency of anti-cancer therapy. In this paper we investigate the existence, uniqueness and growth rate of the solution to a mathematical model of unperturbed human tumour cell line. At first, we construct the solution of this mathematical model by the method of continuation of solution, and then show the solution is unique. Finally, we find that the growth rate of the solution with respect to time is faster than exponential function. The basic mathematical techniques used here are variation of parameters and upper and lower solutions for differential equations. These results allowed one to estimate the cells population in each phase at specific time while one does not have cells mitosis DNA distribution data and it can also be used to compare with the perturbed cell lines.
\end{abstract}

\section{INTRODUCTION}

Cell proliferation is closely associated with cell growth, programmed cell death and tumor formation. The progression lays foundation for cell differentiation. As cells enter mitotic division, the two daughter cells acquire the same genetic information and, as they grow, the heredity propagates from generation to generation. The growth of cells involves DNA duplication before mitosis, which constitutes part of cell cycle. Most of the cells stay at the interphase between the two successive cell divisions. During mitosis, the cell chromosomes undergo DNA duplication first and the tetraploid chromosomes are distributed evenly to two daughter nuclei following mitosis. Cell cycle can be divided into four distinct phases, e.g., $G_{1^{-}}, S-, G_{2^{-}}$and $M$-phases (Fig. 1). DNA replication occurs during synthesis phase or $S$-phase, in which the new chromosome components were formed. The duration between previous mitosis and the initiation of $S$-phase is termed $G_{1}$-phase. The enzymes required

Received May 14, 2010, accepted October 1, 2010.

Communicated by George Yin.

2010 Mathematics Subject Classification: 34, 35, 92.

Key words and phrases: Tumour cell line, Global existence, Upper and lower solutions, Monotone iterations. 
for DNA synthesis appeared at the final stage of $G_{1}$-phase. As cells leave $S$-phase and enter $G_{2}$-phase, an increase of protein assists in cell division. The completion of $G_{2}$-phase marks transition into $M$-phase, i.e. the initiation of mitosis.

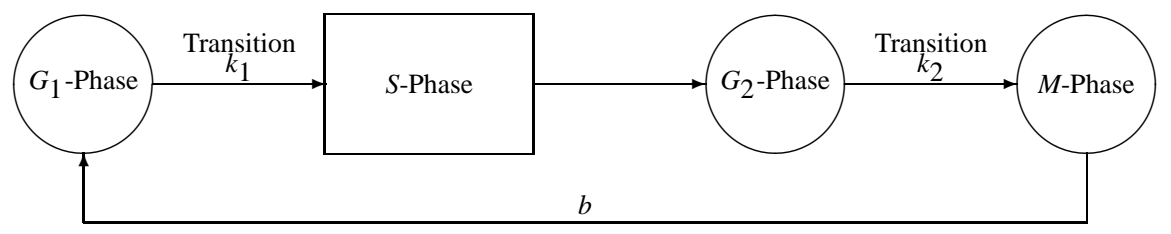

Fig. 1. Cell cycle

This diagram is the phase transition of a cell population. The cell cycle has four distinct phases: $G_{1}{ }^{-}, S_{-}^{-}$, $G_{2}$ - and $M$-phase. Here $k_{1}$ is the hourly rate of transition of cells from $G_{1}$-phase to $S$-phase, $k_{2}$ is the hourly rate of transition of cells from $G_{2}$-phase to $M$-phase and $b$ is the hourly rate of cell division from $M$-phase to $G_{1}$-phase. The square box shaped in the $S$-phase depicts the assumption that cells spend a fixed time ( $T_{S}$ hours) in the $S$-phase.

One way to describe the progression of cell cycle in a cohort of cells is achieved by measuring their DNA variation through flow cytometry. The method involves DNA staining with fluorescent materials and the emitted fluorescence is proportional to cellular DNA contents. The histograms obtained in analyzed cells signify cell phase distributions, in which similar DNA contents at each phase are sorted together.

Anticancer drugs affected growth of cancer cells through programmed cell death (apoptosis) and cell cycle arrest. Due to different sensitivities to the drugs on various cell lines, the characteristic durations at each phase as obtained from DNA histograms by flow cytometry reflect the efficacy after treatment. In this work, we will define "perturbed" as the cancer cells having been exposed to therapy, while "unperturbed" as those unexposed. In order to understand the efficiency of anticancer therapy, it is important to understand the proliferation of unperturbed cells at first. For that purpose, we have developed a mathematical model to analyze and compare cell lines unperturbed by any therapy. This essential step is a prerequisite prior to comparison with the perturbed cell lines, since all designed experiments begin with unperturbed cells. The results from our analysis yield good results with significant implications.

Recently, the mathematical modeling of the cell population was broadly studied (see e.g., [1-13, 20-21, 23-27]). In [2] (2004), Basse and others proposed a mathematical model concerning the dynamics of unperturbed human tumor cell population. This mathematical model involves the following four differential equations:

$$
\frac{\partial}{\partial t} G_{1}(x, t)=4 b M(2 x, t)-k_{1} G_{1}(x, t),
$$




$$
\begin{aligned}
& \frac{\partial}{\partial \tau} \bar{S}(x, t ; \tau)+\frac{\partial}{\partial t} \bar{S}(x, t ; \tau)=D \frac{\partial^{2}}{\partial x^{2}} \bar{S}(x, t ; \tau)-g_{S} \frac{\partial}{\partial x} \bar{S}(x, t ; \tau), \\
& \frac{\partial}{\partial t} G_{2}(x, t)=\bar{S}\left(x, t ; T_{S}\right)-k_{2} G_{2}(x, t), \\
& \frac{\partial}{\partial t} M(x, t)=k_{2} G_{2}(x, t)-b M(x, t),
\end{aligned}
$$

which accompany with the following initial and boundary conditions:

$$
\begin{aligned}
& G_{1}(x, 0)=G_{1,0}(x), \quad 0 \leq x \leq L, \\
& \bar{S}(x, 0 ; \tau)=\bar{S}_{0}(x ; \tau), \quad 0 \leq \tau \leq T_{S}, \quad 0 \leq x \leq L, \\
& \bar{S}(x, t ; 0)=k_{1} G_{1}(x, t), \quad t \geq 0, \quad 0 \leq x \leq L, \\
& D \frac{\partial}{\partial x} \bar{S}(0, t ; \tau)-g_{S} \bar{S}(0, t ; \tau)=0, \quad t>0, \quad 0<\tau \leq T_{S}, \\
& D \frac{\partial}{\partial x} \bar{S}(L, t ; \tau)-g_{S} \bar{S}(L, t ; \tau)=0, \quad t>0, \quad 0<\tau \leq T_{S}, \\
& G_{2}(x, 0)=G_{2,0}(x), \quad 0 \leq x \leq L, \\
& M(x, 0)=M_{0}(x), \quad 0 \leq x \leq L .
\end{aligned}
$$

More precisely, $G_{1}(x, t)$ represents the number density of cells in $G_{1}$-phase at time $t>0$ (hours) where $0 \leq x \leq L$ is the relative DNA content (dimensionless) and $L$ is the maximum DNA content. The number density of cells that have been in the $S$-phase for $\tau$ hours at time $t$ is $\bar{S}(x, t ; \tau)$ and $S(x, t)=\int_{0}^{T_{S}} \bar{S}(x, t ; \tau) d \tau$, while the number densities of cells in the $G_{2}$-phase and $M$-phase are $G_{2}(x, t)$ and $M(x, t)$, respectively. Model parameters with their descriptions are the following:

$k_{1}$ is the hourly rate of transition between the $G_{1^{-}}$and the $S$-phase;

$D$ is the dispersion coefficient;

$g_{S}$ is the rate of DNA increase per hour in $S$-phase;

$T_{S}$ is the time in $S$-phase;

$k_{2}$ is the hourly rate of transition between the $G_{2^{-}}$and the $M$-phase;

$b$ is the hourly rate of division from the $M$-phase.

In [5], by integrating the differential equations (with boundary conditions) with respect to $x$ from 0 to $L$, they proposed a new unperturbed tumor cell population mathematical model to describe the population functions of cells in $G_{1^{-}}, S-, G_{2^{-}}$ and $M$-phases respectively as follows:

$$
\begin{gathered}
\frac{d}{d t} n_{1}(t)=2 b n_{M}(t)-k_{1} n_{1}(t), \\
\frac{\partial}{\partial \tau} n_{S}(t, \tau)+\frac{\partial}{\partial t} n_{S}(t, \tau)=0,
\end{gathered}
$$




$$
\begin{aligned}
& \frac{d}{d t} n_{2}(t)=n_{S}\left(t, T_{S}\right)-k_{2} n_{2}(t), \\
& \frac{d}{d t} n_{M}(t)=k_{2} n_{2}(t)-b n_{M}(t),
\end{aligned}
$$

where $t>0,0<\tau_{S} \leq T_{S}, n_{1}(t)=\int_{0}^{L} G_{1}(x, t) d x, n_{S}(t, \tau)=\int_{0}^{L} \bar{S}(x, t ; \tau) d x$, $n_{2}(t)=\int_{0}^{L} G_{2}(x, t) d x$ and $n_{M}(t)=\int_{0}^{L} M(x, t) d x$. The functions $n_{1}(t), n_{2}(t)$ and $n_{M}(t)$ represent the tumour cell populations in $G_{1^{-}}, G_{2^{-}}$and $M$-phases at time $t$, respectively; and the function $n_{S}(t, \tau)$ represents the tumour cell population in $S$-phase at time $t$ for them already stated in there for $\tau$ hours. In equation (1.1) $n_{M}(t)=\int_{0}^{2 L} M(x, t) d x$ while in equation (1.4) $n_{M}(t)=\int_{0}^{L} M(x, t) d x$, which is based on the fact that the cell fission only occurred while the DNA content has duplicated. They assumed that the maximum DNA content $L$ is chosen large enough so that the two integrals are equal (that is $\int_{0}^{2 L} M(x, t) d x=\int_{0}^{L} M(x, t) d x$ ). Since $n_{1}(t)$ represents the tumour cell population in $G_{1}$-phase at time $t, n_{S}(t, \tau)$ represents the tumour cell population in $S$-phase at time $t$ for them already stated in there for $\tau$ hours and $k_{1}$ represent the hourly rate of transition from $G_{1}$-phase to $S$ phase, these imply that $n_{S}(t, 0)=k_{1} n_{1}(t)$ for $\tau=0$. Thus, the initial condition in the $S$-phase for equation (1.2) corresponding to $\tau=0$ is $n_{S}(t, 0)=k_{1} n_{1}(t)$. The initial conditions in each phases corresponding to $t=0$ are obtained by integration over $x$ of the initial DNA distributions $G_{1,0}(x), \bar{S}_{0}(x ; \tau), G_{2,0}(x)$ and $M_{0}(x)$, respectively; i.e., the initial conditions of cells population functions in each phases are given by:

$$
\begin{gathered}
n_{1,0}=n_{1}(0)=\int_{0}^{L} G_{1,0}(x) d x \\
n_{S, 0}(\tau)=n_{S}(0, \tau)=\int_{0}^{L} \bar{S}_{0}(x, \tau) d x, \quad 0 \leq \tau \leq T_{S}, \\
n_{2,0}=n_{2}(0)=\int_{0}^{L} G_{2,0}(x) d x \\
n_{M, 0}=n_{M}(0)=\int_{0}^{L} M_{0}(x) d x \\
n_{S}(t ; 0)=k_{1} n_{1}(t), \quad t \geq 0
\end{gathered}
$$

Since both $n_{S}(t, \tau)$ and $n_{1}(t)$ are continuous functions, we have

$$
n_{S, 0}(0)=n_{S}(0,0)=\lim _{t \rightarrow 0} n_{S}(t, 0)=\lim _{t \rightarrow 0} k_{1} n_{1}(t)=k_{1} n_{1,0} .
$$

In [5], they used finite difference method with Matlab as a tool to get the numerical solution of this differential system; they also compared the solution with the experimental data to illustrate the validity of this mathematical model. This human tumor cell mathematical model allowed one to estimate the cells population in each 
phase at specific time while one does not have cells mitosis DNA distribution data. Hence one can obtain the flow cytometric profile at any particular time (see e.g., [27]) and study the problem of the steady DNA distribution (so called SDD problem) for human tumor cell's DNA. Furthermore, one can extrapolate the efficiency of the anticancer therapy.

In section 2, we are trying to find the representations of the solutions of (1.1)(1.9) in terms of closed forms. At first, we declare without proof a well-known result that the solutions of the differential system (1.1)-(1.4) are also the solutions of an integral system (2.1)-(2.4) (please see theorem 2.1), and then we apply the continuation of the solution method to construct the solutions of the integral system. We proved the uniqueness of the solutions of the integral system in theorem 2.2. Under the assumption $n_{S, 0}(\tau)$ to be a continuously differentiable function on $\left[0, T_{S}\right]$ with $n_{S, 0}(0)=k_{1} n_{1,0}$, we prove that the solution of the integral system (2.1)-(2.4) is also the solution of the differential system (1.1)-(1.9) except possibly on the line $t=\tau$ (please see Theorem 2.3). At the end of section 2, we show that if the initial function $n_{S, 0}$ satisfying the condition $\frac{d}{d \tau} n_{S, 0}(0)=k_{1}^{2} n_{1,0}-2 b k_{1} n_{M, 0}$ then the solution of the integral system (2.1)-(2.4) is also the solution the differential system (1.1)-(1.9) (please see Theorem 2.4). However, this condition just comes from the mathematical point of view.

If one understands the populations of unperturbed cell lines at a certain time, he can follow the procedure in the proof of Theorem 2.2 to compute the unperturbed cell populations in each phase at any time afterwards. From the closed form of unperturbed cell line population functions

$$
\begin{aligned}
& n_{1}(t)=\exp \left(-k_{1} t\right) n_{1,0}+2 b \int_{0}^{t} \exp \left(-k_{1}(t-\eta)\right) n_{M}(\eta) d \eta, \\
& n_{S}(t, \tau)=\left\{\begin{array}{cc}
n_{S, 0}(\tau-t) & \text { if } T_{S} \geq \tau \geq t \geq 0, \\
k_{1} n_{1}(t-\tau) & \text { if } \infty>t \geq \tau \geq 0,
\end{array}\right. \\
& n_{2}(t)=\exp \left(-k_{2} t\right) n_{2,0}+\int_{0}^{t} \exp \left(-k_{2}(t-\eta)\right) n_{S}\left(\eta, T_{S}\right) d \eta, \\
& n_{M}(t)=\exp (-b t) n_{M, 0}+k_{2} \int_{0}^{t} \exp (-b(t-\eta)) n_{2}(\eta) d \eta,
\end{aligned}
$$

for all $t \in[0, \infty)$ and $\tau \in\left[0, T_{S}\right]$, the populations in each phase were depending on the initial functions and transition coefficients, respectively. The work explains why the populations in each phase of different unperturbed cell lines are distributed differently. Furthermore, if the populations of human tumor cells in each of $G_{1^{-}}$, $S-, G_{2^{-}}$and $M$-phase of unperturbed cells at a certain time can be understood, it is then predictable how those in each phase of unperturbed cells be like. The result can be further extended to compare with the phase distributions in tumor cells under anticancer treatments and hence to predict their efficiency. 
In Section 3, we are going to estimate the growth rate of the unperturbed cell line populations in each phase. For achieving this goal, we apply the upper and lower solutions method. We first show that if $\left\{\left(\bar{n}_{1}^{(m)}, \bar{n}_{S}^{(m)}, \bar{n}_{2}^{(m)}, \bar{n}_{M}^{(m)}\right)\right\}$ and $\left\{\left(\underline{n}_{1}^{(m)}, \underline{n}_{S}^{(m)}, \underline{n}_{2}^{(m)}, \underline{n}_{M}^{(m)}\right)\right\}, m \in \mathbb{N}$ are maximal and minimal sequences for this mathematical model, then they possess the monotone property $\widehat{n}_{i} \leq \underline{n}_{i}^{(m)} \leq$ $\underline{n}_{i}^{(m+1)} \leq \bar{n}_{i}^{(m+1)} \leq \bar{n}_{i}^{(m)} \leq \widetilde{n}_{i}(i=1,2, S$ or $M)$ for all $m \in \mathbb{N}$. We prove the maximal and the minimal sequences converge monotonically from above and below respectively to a unique solution $\left(n_{1}, n_{S}, n_{2}, n_{M}\right)$ of the system (1.1)-(1.4) (please see Lemmas 3.1-3.3 and Theorem 3.1). In general, as long as the tumour cell is observed, the populations in each phase are positive. So, we may assume that $n_{1}(0)>0, \min _{\tau \in\left[0, T_{S}\right]} n_{S}(0, \tau)>0, n_{2}(0)>0$ and $n_{M}(0)>0$. Under these assumptions, we find the functions

$$
\widehat{n}_{1}(t)=\frac{\rho}{k_{1}} e^{\epsilon t}, \widehat{n}_{S}(t, \tau)=\rho e^{\epsilon(t-\tau)}, \widehat{n}_{2}(t)=\frac{(1+\delta)^{2}}{2 k_{2}} \rho e^{\epsilon t} \text { and } \widehat{n}_{M}(t)=\frac{1+\delta}{2 b} \rho e^{\epsilon t}
$$

are lower solutions for the system (1.1)-(1.4), and hence the population functions satisfy

$$
n_{1}(t) \geq \frac{\rho}{k_{1}} e^{\epsilon t}, n_{S}(t, \tau) \geq \rho e^{\epsilon(t-\tau)}, n_{2}(t) \geq \frac{(1+\delta)^{2}}{2 k_{2}} \rho e^{\epsilon t} \text { and } n_{M}(t) \geq \frac{1+\delta}{2 b} \rho e^{\epsilon t}
$$

for all $t \in[0, \infty)$ and all $\tau \in\left[0, T_{S}\right]$. In these inequalities, $\delta, \varepsilon$ and $\rho$ are positive constants depending on the initial functions and transition coefficients (please see Theorem 3.2). Thus the growth rate of the population in each of the $G_{1^{-}}, S-, G_{2^{-}}$ and $M$-phase of unperturbed cells is faster than an exponential function.

The results of section 2 and section 3 can be further extended to compare with the phase distributions in tumor cells under anticancer treatments and hence to predict their efficiency (please see the last section).

\section{EXISTENCE AND UNIQUENESS}

In this section we show the existence and uniqueness of a solution of the initialboundary value problem (1.1)-(1.9). We start with the statement of a well-known result that a solution of the differential system is also the solution of a corresponding integral system. However, the proof of this theorem is standard and straight forward; we left the details of the proof for the interesting reader.

Theorem 2.1. If $\left(n_{1}(t), n_{S}(t, \tau), n_{2}(t), n_{M}(t)\right)$ is the solution of the initialboundary value problem (1.1)-(1.9) with the initial condition $\left(n_{1,0}, n_{S, 0}(\tau), n_{2,0}\right.$, $\left.n_{M, 0}\right)$ on $[0, \infty) \times\left[0, T_{S}\right]$, then the solution $\left(n_{1}(t), n_{S}(t, \tau), n_{2}(t), n_{M}(t)\right)$ satisfies the following integral system:

$$
n_{1}(t)=\exp \left(-k_{1} t\right) n_{1,0}+2 b \int_{0}^{t} \exp \left(-k_{1}(t-\eta)\right) n_{M}(\eta) d \eta
$$




$$
\begin{gathered}
n_{S}(t, \tau)=\left\{\begin{array}{cc}
n_{S, 0}(\tau-t) & \text { if } T_{S} \geq \tau \geq t \geq 0, \\
k_{1} n_{1}(t-\tau) & \text { if } \infty>t \geq \tau \geq 0,
\end{array}\right. \\
n_{2}(t)=\exp \left(-k_{2} t\right) n_{2,0}+\int_{0}^{t} \exp \left(-k_{2}(t-\eta)\right) n_{S}\left(\eta, T_{S}\right) d \eta, \\
n_{M}(t)=\exp (-b t) n_{M, 0}+k_{2} \int_{0}^{t} \exp (-b(t-\eta)) n_{2}(\eta) d \eta .
\end{gathered}
$$

We prove that the integral system (2.1)-(2.4) has a unique solution $\left(n_{1}(t), n_{S}\right.$ $\left.(t, \tau), n_{2}(t), n_{M}(t)\right)$.

Theorem 2.2. Suppose $n_{1,0}, n_{2,0}, n_{M, 0} \in \mathbb{R}$, and $n_{S, 0}(\tau)$ is a continuous function on $\left[0, T_{S}\right]$ with $n_{S, 0}(0)=k_{1} n_{1,0}$. Then the system (2.1)-(2.4) has a unique solution $\left(n_{1}(t), n_{S}(t, \tau), n_{2}(t), n_{M}(t)\right)$.

Proof. To construct a solution of the integral system (2.1)-(2.4), we divide the interval $[0, \infty)$ into subintervals $\left[(n-1) T_{S}, n T_{S}\right]$ for all $n \in \mathbb{N}$. Firstly, we consider the case that $(t, \tau) \in\left[0, T_{S}\right] \times\left[0, T_{S}\right]$. Let $u_{1,2}:\left[0, T_{S}\right] \rightarrow \mathbb{R}$ be defined by

$$
u_{1,2}(t)=\exp \left(-k_{2} t\right) n_{2,0}+\int_{0}^{t} \exp \left(-k_{2}(t-\eta)\right) n_{S, 0}\left(T_{S}-\eta\right) d \eta .
$$

From assumption, $u_{1,2}(t)$ is a continuous function on $\left[0, T_{S}\right]$. Thus we can subsequently define functions $u_{1, M}:\left[0, T_{S}\right] \rightarrow \mathbb{R}, u_{1,1}:\left[0, T_{S}\right] \rightarrow \mathbb{R}$ and $u_{1, S}:$ $\left[0, T_{S}\right] \times\left[0, T_{S}\right] \rightarrow \mathbb{R}$ by

$$
\begin{aligned}
u_{1, M}(t) & =\exp (-b t) n_{M, 0}+k_{2} \int_{0}^{t} \exp (-b(t-\eta)) u_{1,2}(\eta) d \eta, \\
u_{1,1}(t) & =\exp \left(-k_{1} t\right) n_{1,0}+2 b \int_{0}^{t} \exp \left(-k_{1}(t-\eta)\right) u_{1, M}(\eta) d \eta, \\
u_{1, S}(t, \tau) & = \begin{cases}n_{S, 0}(\tau-t) & \text { if } T_{S} \geq \tau \geq t \geq 0, \\
k_{1} u_{1,1}(t-\tau) & \text { if } T_{S} \geq t \geq \tau \geq 0 .\end{cases}
\end{aligned}
$$

Obviously, $u_{1, M}(t)$ and $u_{1,1}(t)$ are continuous functions on $\left[0, T_{S}\right]$. Since both $n_{S, 0}$ and $k_{1} u_{1,1}$ are continuous in their domain, and

$$
\lim _{\tau-t \rightarrow 0^{+}} n_{S, 0}(\tau-t)=n_{S, 0}(0)=k_{1} n_{1,0}=\lim _{t-\tau \rightarrow 0^{+}} k_{1} u_{1,1}(t-\tau),
$$

$u_{1, S}(t, \tau)$ is also a continuous function on $\left[0, T_{S}\right] \times\left[0, T_{S}\right]$. According to this definition, $u_{1, S}\left(\eta, T_{S}\right)=n_{S, 0}\left(T_{S}-\eta\right)$ provided that $T_{S} \geq t \geq \eta \geq 0$ and hence

$$
u_{1,2}(t)=\exp \left(-k_{2} t\right) n_{2,0}+\int_{0}^{t} \exp \left(-k_{2}(t-\eta)\right) u_{1, S}\left(\eta, T_{S}\right) d \eta
$$


for all $T_{S} \geq t \geq \eta \geq 0$. These imply that $\left(u_{1,1}(t), u_{1, S}(t, \tau), u_{1,2}(t), u_{1, M}(t)\right)$ is a solution of the system (2.1)-(2.4).

Secondary, we consider the case that $(t, \tau) \in\left[T_{S}, 2 T_{S}\right] \times\left[0, T_{S}\right]$. Let $u_{2,2}$ : $\left[T_{S}, 2 T_{S}\right] \rightarrow \mathbb{R}$ be defined by

$$
u_{2,2}(t)=e^{-k_{2}\left(t-T_{S}\right)} u_{1,2}\left(T_{S}\right)+k_{1} \int_{0}^{t-T_{S}} \exp \left(-k_{2}\left(t-T_{S}-\eta\right)\right) u_{1,1}(\eta) d \eta
$$

Since $u_{1,1}(t)$ is a continuous function on $\left[0, T_{S}\right], u_{2,2}(t)$ is a continuous function on $\left[T_{S}, 2 T_{S}\right]$. Set functions $u_{2, M}:\left[T_{S}, 2 T_{S}\right] \rightarrow \mathbb{R}, u_{2,1}:\left[T_{S}, 2 T_{S}\right] \rightarrow \mathbb{R}$ and $u_{2, S}:\left[T_{S}, 2 T_{S}\right] \times\left[0, T_{S}\right] \rightarrow \mathbb{R}$ subsequently by

$$
\begin{gathered}
u_{2, M}(t)=\exp \left(-b\left(t-T_{S}\right)\right) u_{1, M}\left(T_{S}\right)+k_{2} \int_{T_{S}}^{t} \exp (-b(t-\eta)) u_{2,2}(\eta) d \eta, \\
u_{2,1}(t)=\exp \left(-k_{1}\left(t-T_{S}\right)\right) u_{1,1}\left(T_{S}\right)+2 b \int_{T_{S}}^{t} \exp \left(-k_{1}(t-\eta)\right) u_{2, M}(\eta) d \eta, \\
u_{2, S}(t, \tau)= \begin{cases}k_{1} u_{1,1}(t-\tau) & \text { if } T_{S}+\tau \geq t \geq T_{S}, \\
k_{1} u_{2,1}(t-\tau) & \text { if } 2 T_{S} \geq t \geq T_{S}+\tau .\end{cases}
\end{gathered}
$$

respectively. Then $u_{2, M}(t)$ and $u_{2,1}(t)$ are continuous functions on $\left[T_{S}, 2 T_{S}\right]$. Moreover, since both $k_{1} u_{1,1}$ and $k_{1} u_{2,1}$ are continuous in their domain, and

$$
\lim _{t-\tau \rightarrow T_{S}^{-}} u_{1,1}(t-\tau)=u_{1,1}\left(T_{S}\right)=u_{2,1}\left(T_{S}\right)=\lim _{t-\tau \rightarrow T_{S}{ }^{+}} u_{2,1}(t-\tau),
$$

$u_{2, S}(t, \tau)$ is also a continuous function on $\left[T_{S}, 2 T_{S}\right] \times\left[0, T_{S}\right]$. Let functions $n_{1}$ : $\left[0,2 T_{S}\right] \rightarrow \mathbb{R}, n_{2}:\left[0,2 T_{S}\right] \rightarrow \mathbb{R}, n_{M}:\left[0,2 T_{S}\right] \rightarrow \mathbb{R}$ and $n_{S}:\left[0,2 T_{S}\right] \times\left[0, T_{S}\right] \rightarrow$ $\mathbb{R}$ be defined by

$$
\begin{aligned}
n_{1}(t) & = \begin{cases}u_{1,1}(t) & \text { if } 0 \leq t \leq T_{S}, \\
u_{2,1}(t) & \text { if } T_{S} \leq t \leq 2 T_{S}\end{cases} \\
n_{2}(t) & = \begin{cases}u_{1,2}(t) & \text { if } 0 \leq t \leq T_{S}, \\
u_{2,2}(t) & \text { if } T_{S} \leq t \leq 2 T_{S} ;\end{cases} \\
n_{M}(t) & = \begin{cases}u_{1, M}(t) & \text { if } 0 \leq t \leq T_{S}, \\
u_{2, M}(t) & \text { if } T_{S} \leq t \leq 2 T_{S} ;\end{cases} \\
n_{S}(t, \tau) & = \begin{cases}u_{1, S}(t, \tau) & \text { if } 0 \leq t \leq T_{S}, 0 \leq \tau \leq T_{S}, \\
u_{2, S}(t, \tau) & \text { if } T_{S} \leq t \leq 2 T_{S}, 0 \leq \tau \leq T_{S} .\end{cases}
\end{aligned}
$$

Then they are solutions of (2.1)-(2.4). In fact, from the definitions of these functions, we have 


$$
\begin{aligned}
n_{M}(t) & =u_{1, M}(t)=\exp (-b t) n_{M, 0}+k_{2} \int_{0}^{t} \exp (-b(t-\eta)) u_{1,2}(\eta) d \eta \\
& =e^{-b t} n_{M, 0}+k_{2} \int_{0}^{t} e^{-b(t-\eta)} n_{2}(\eta) d \eta \quad \text { for all } 0 \leq t \leq T_{S},
\end{aligned}
$$

and

$$
\begin{aligned}
n_{M}(t) & =u_{2, M}(t) \\
& =\exp \left(-b\left(t-T_{S}\right)\right) u_{1, M}\left(T_{S}\right)+k_{2} \int_{T_{S}}^{t} \exp (-b(t-\eta)) u_{2,2}(\eta) d \eta \\
& =e^{-b\left(t-T_{S}\right)}\left[e^{-b T_{S}} n_{M, 0}+k_{2} \int_{0}^{T_{S}} e^{-b\left(T_{S}-\eta\right)} u_{1,2}(\eta) d \eta\right] \\
& +k_{2} \int_{T_{S}}^{t} e^{-b(t-\eta)} u_{2,2}(\eta) d \eta \\
& =e^{-b t} n_{M, 0}+k_{2} \int_{0}^{T_{S}} e^{-b(t-\eta)} n_{2}(\eta) d \eta+k_{2} \int_{T_{S}}^{t} e^{-b(t-\eta)} n_{2}(\eta) d \eta \\
& =e^{-b t} n_{M, 0}+k_{2} \int_{0}^{t} e^{-b(t-\eta)} n_{2}(\eta) d \eta \text { for all } T_{S} \leq t \leq 2 T_{S} .
\end{aligned}
$$

Thus,

$n_{M}(t)=\exp (-b t) n_{M, 0}+k_{2} \int_{0}^{t} \exp (-b(t-\eta)) n_{2}(\eta) d \eta$ for all $t \in\left[0,2 T_{S}\right]$.

Similarly, we have

$$
n_{1}(t)=e^{-k_{1} t} n_{1,0}+2 b \int_{0}^{t} e^{-k_{1}(t-\eta)} n_{M}(\eta) d \eta \text { for all } t \in\left[0,2 T_{S}\right],
$$

and

$$
\begin{aligned}
n_{2}(t) & =u_{1,2}(t)=\exp \left(-k_{2} t\right) n_{2,0}+\int_{0}^{t} \exp \left(-k_{2}(t-\eta)\right) u_{1, S}\left(\eta, T_{S}\right) d \eta \\
& =e^{-k_{2} t} n_{2,0}+\int_{0}^{t} e^{-k_{2}(t-\eta)} n_{S}\left(\eta, T_{S}\right) d \eta \quad \text { for all } 0 \leq t \leq T_{S} .
\end{aligned}
$$

Since $n_{S}\left(\eta, T_{S}\right)=u_{2, S}\left(\eta, T_{S}\right)=k_{1} u_{1,1}\left(\eta-T_{S}\right)$ as long as $2 T_{S} \geq t \geq \eta \geq$ $T_{S}$, this implies that

$$
\begin{aligned}
n_{2}(t) & =u_{2,2}(t) \\
& =e^{-k_{2}\left(t-T_{S}\right)} u_{1,2}\left(T_{S}\right)+k_{1} \int_{0}^{t-T_{S}} e^{-k_{2}\left(t-T_{S}-\eta\right)} u_{1,1}(\eta) d \eta \\
& =e^{-k_{2}\left(t-T_{S}\right)} n_{2}\left(T_{S}\right)+k_{1} \int_{T_{S}}^{t} e^{-k_{2}(t-\eta)} u_{1,1}\left(\eta-T_{S}\right) d \eta
\end{aligned}
$$




$$
\begin{aligned}
& =e^{-k_{2} t} n_{2,0}+\int_{0}^{T_{S}} e^{-k_{2}(t-\eta)} n_{S}\left(\eta, T_{S}\right) d \eta+\int_{T_{S}}^{t} e^{-k_{2}(t-\eta)} n_{S}\left(\eta, T_{S}\right) d \eta \\
& =\exp \left(-k_{2} t\right) n_{2,0}+\int_{0}^{t} \exp \left(-k_{2}(t-\eta)\right) n_{S}\left(\eta, T_{S}\right) d \eta
\end{aligned}
$$

for all $T_{S} \leq t \leq 2 T_{S}$. Now, we consider the function $n_{S}(t, \tau)$ on the region $\left[0,2 T_{S}\right] \times\left[0, T_{S}\right]$. As $(t, \tau)$ in the region $\left[0, T_{S}\right] \times\left[0, T_{S}\right]$, we have

$$
n_{S}(t, \tau)=u_{1, S}(t, \tau)= \begin{cases}n_{S, 0}(\tau-t) & \text { if } \quad T_{S} \geq \tau \geq t \geq 0, \\ k_{1} u_{1,1}(t-\tau)=k_{1} n_{1}(t-\tau) & \text { if } \quad T_{S} \geq t \geq \tau \geq 0 .\end{cases}
$$

As $(t, \tau)$ in the region $\left[T_{S}, 2 T_{S}\right] \times\left[0, T_{S}\right]$, then either $T_{S}+\tau \geq t \geq T_{S}$ or $2 T_{S} \geq t \geq T_{S}+\tau$. If $T_{S}+\tau \geq t \geq T_{S}$, that is $T_{S} \geq t-\tau \geq 0$, then

$$
n_{S}(t, \tau)=u_{2, S}(t, \tau)=k_{1} u_{1,1}(t-\tau)=k_{1} n_{1}(t-\tau) .
$$

On the other hand, if $2 T_{S} \geq t \geq T_{S}+\tau$, that is $2 T_{S} \geq t-\tau \geq T_{S}$, then

$$
n_{S}(t, \tau)=u_{2, S}(t, \tau)=k_{1} u_{2,1}(t-\tau)=k_{1} n_{1}(t-\tau) .
$$

These imply that

$$
n_{S}(t, \tau)= \begin{cases}n_{S, 0}(\tau-t) & \text { if } T_{S} \geq \tau \geq t \geq 0, \\ k_{1} n_{1}(t-\tau) & \text { if } 2 T_{S} \geq t \geq \tau \geq 0 .\end{cases}
$$

With a similar argument, one can extend $\left(n_{1}(t), n_{S}(t, \tau), n_{2}(t), n_{M}(t)\right)$ to a global solution of the system (2.1)-(2.4).

This theorem will be proved as long as we show that $\left(n_{1}(t), n_{S}(t, \tau), n_{2}(t)\right.$, $\left.n_{M}(t)\right)$ is the unique solution of (2.1)-(2.4). Suppose that $\left(v_{1}(t), v_{S}(t, \tau), v_{2}(t)\right.$, $\left.v_{M}(t)\right)$ is a solution of the system (2.1)-(2.4), then they satisfy

$$
\begin{aligned}
& v_{1}(t)=\exp \left(-k_{1} t\right) n_{1,0}+2 b \int_{0}^{t} \exp \left(-k_{1}(t-\eta)\right) v_{M}(\eta) d \eta, \\
& v_{S}(t, \tau)= \begin{cases}n_{S, 0}(\tau-t) & \text { if } T_{S} \geq \tau \geq t \geq 0, \\
k_{1} v_{1}(t-\tau) & \text { if } \quad \infty>t \geq \tau \geq 0,\end{cases} \\
& v_{2}(t)=\exp \left(-k_{2} t\right) n_{2,0}+\int_{0}^{t} \exp \left(-k_{2}(t-\eta)\right) v_{S}\left(\eta, T_{S}\right) d \eta, \\
& v_{M}(t)=\exp (-b t) n_{M, 0}+k_{2} \int_{0}^{t} \exp (-b(t-\eta)) v_{2}(\eta) d \eta
\end{aligned}
$$


From the closed forms of $n_{1}(t), n_{M}(t), v_{1}(t), v_{M}(t)$, we have

$$
\left|n_{1}(t)-v_{1}(t)\right| \leq 2 b \int_{0}^{t} \exp \left(-k_{1}(t-\eta)\right)\left|n_{M}(\eta)-v_{M}(\eta)\right| d \eta,
$$

and

$$
\left|n_{M}(t)-v_{M}(t)\right| \leq k_{2} \int_{0}^{t} \exp (-b(t-\eta))\left|n_{2}(\eta)-v_{2}(\eta)\right| d \eta .
$$

Since

$$
\begin{aligned}
v_{2}(t)= & \exp \left(-k_{2} t\right) n_{2,0}+\int_{0}^{t} \exp \left(-k_{2}(t-\eta)\right) v_{S}\left(\eta, T_{S}\right) d \eta \\
= & e^{-k_{2} t} n_{2,0}+\int_{0}^{T_{S}} e^{-k_{2}(t-\eta)} n_{S, 0}\left(T_{S}-\eta\right) d \eta \\
& +k_{1} \int_{T_{S}}^{t} e^{-k_{2}(t-\eta)} v_{1}\left(\eta-T_{S}\right) d \eta \\
= & e^{-k_{2} t} n_{2,0}+\int_{0}^{T_{S}} e^{-k_{2}(t-\eta)} n_{S, 0}\left(T_{S}-\eta\right) d \eta \\
& +k_{1} e^{k_{2} T_{S}} \int_{0}^{t-T_{S}} e^{-k_{2}(t-\eta)} v_{1}(\eta) d \eta,
\end{aligned}
$$

we have

$$
\begin{aligned}
& \left|n_{2}(t)-v_{2}(t)\right| \leq k_{1} e^{k_{2} T_{S}} \int_{0}^{t-T_{S}} \exp \left(-k_{2}(t-\eta)\right)\left|n_{1}(\eta)-v_{1}(\eta)\right| d \eta \\
\leq & k_{1} e^{k_{2} T_{S}} \int_{0}^{t} \exp \left(-k_{2}(t-\eta)\right)\left|n_{1}(\eta)-v_{1}(\eta)\right| d \eta .
\end{aligned}
$$

Let $K=\max \left\{k_{1} e^{k_{2} T_{S}}, k_{2}, 2 b\right\}, \alpha=\min \left\{k_{1}, k_{2}, b\right\}$ and $g(t)=\left|n_{1}(t)-v_{1}(t)\right|+\left|n_{2}(t)-v_{2}(t)\right|+\left|n_{M}(t)-v_{M}(t)\right|$ forall $t \in[0, \infty)$.

Then

$$
\begin{aligned}
g(t) \leq & 2 b \int_{0}^{t} e^{-k_{1}(t-\eta)}\left|n_{M}(\eta)-v_{M}(\eta)\right| d \eta \\
& +k_{1} e^{k_{2} T_{S}} \int_{0}^{t} e^{-k_{2}(t-\eta)}\left|n_{1}(\eta)-v_{1}(\eta)\right| d \eta \\
& +k_{2} \int_{0}^{t} e^{-b(t-\eta)}\left|n_{2}(\eta)-v_{2}(\eta)\right| d \eta \\
\leq & K \int_{0}^{t} e^{-\alpha(t-\eta)}\left(\left|n_{M}(\eta)-v_{M}(\eta)\right|\right. \\
& \left.+\left|n_{1}(\eta)-v_{1}(\eta)\right|+\left|n_{2}(\eta)-v_{2}(\eta)\right|\right) d \eta \\
= & K \int_{0}^{t} e^{-\alpha(t-\eta)} g(\eta) d \eta \quad \text { forall } t \in[0, \infty) .
\end{aligned}
$$


This implies that $e^{\alpha t} g(t) \leq K \int_{0}^{t} e^{\alpha \eta} g(\eta) d \eta$ for all $t \in[0, \infty)$. By Gronwall's inequality $e^{\alpha t} g(t)=0$ for all $t \in[0, \infty)$, and hence

$$
g(t)=\left|n_{1}(t)-v_{1}(t)\right|+\left|n_{2}(t)-v_{2}(t)\right|+\left|n_{M}(t)-v_{M}(t)\right|=0
$$

for all $t \in[0, \infty)$. Therefore $n_{1}(t)=v_{1}(t), n_{2}(t)=v_{2}(t)$ and $n_{M}(t)=v_{M}(t)$ for all $t \in[0, \infty)$. These imply that $n_{S}(t, \tau)=v_{S}(t, \tau)$ on $[0, \infty) \times\left[0, T_{S}\right]$, and the proof of this theorem is complete.

We will end this section by proving the solution $\left(n_{1}(t), n_{S}(t, \tau), n_{2}(t), n_{M}(t)\right)$ of the integral system (2.1)-(2.4) satisfies the differential equations (1.1) and (1.3)(1.9), and $n_{S}(t, \tau)$ satisfies the differential equation (1.2) for all $(t, \tau) \in[0, \infty) \times$ $\left[0, T_{S}\right]$ except possibly on the line $t=\tau$. Furthermore, we will show that if the initial condition $\left(n_{1,0}, n_{S, 0}(\tau), n_{2,0}, n_{M, 0}\right)$ satisfies that $n_{1,0}, n_{2,0}, n_{M, 0} \in \mathbb{R}$, and $n_{S, 0}(\tau)$ is a continuously differentiable function on $\left[0, T_{S}\right]$ with $n_{S, 0}(0)=k_{1} n_{1,0}$ and $n_{S, 0}^{\prime}(0)=k_{1}^{2} n_{1,0}-2 b k_{1} n_{M, 0}$, then the solution satisfies the initial-boundary value problem (1.1)-(1.9). In fact, we have following two theorems.

Theorem 2.3. Suppose $n_{1,0}, n_{2,0}, n_{M, 0} \in \mathbb{R}$, and $n_{S, 0}(\tau)$ is a continuously differentiable function on $\left[0, T_{S}\right]$ with $n_{S, 0}(0)=k_{1} n_{1,0}$, then the solution $\left(n_{1}(t), n_{S}(t, \tau), n_{2}(t), n_{M}(t)\right)$ of the system (2.1)-(2.4) satisfies the differential system (1.1), (1.3)-(1.9), and $n_{S}(t, \tau)$ satisfies the differential equation (1.2) except possibly on the line $t=\tau$.

Proof. Since $\left(n_{1}(t), n_{S}(t, \tau), n_{2}(t), n_{M}(t)\right)$ is the unique solution of the system (2.1)-(2.4), we see that $n_{M}(t), n_{S}\left(t, T_{S}\right), n_{2}(t)$ are continuous on $[0, \infty)$ and

$$
\begin{aligned}
n_{1}(t) & =\exp \left(-k_{1} t\right) n_{1,0}+2 b \int_{0}^{t} \exp \left(-k_{1}(t-\eta)\right) n_{M}(\eta) d \eta, \\
n_{2}(t) & =\exp \left(-k_{2} t\right) n_{2,0}+\int_{0}^{t} \exp \left(-k_{2}(t-\eta)\right) n_{S}\left(\eta, T_{S}\right) d \eta, \\
n_{M}(t) & =\exp (-b t) n_{M, 0}+k_{2} \int_{0}^{t} \exp (-b(t-\eta)) n_{2}(\eta) d \eta .
\end{aligned}
$$

Obviously, $n_{1}(t), n_{2}(t), n_{M}(t)$ are continuously differentiable on $[0, \infty)$. In fact, we have

$$
\begin{aligned}
\frac{d}{d t} n_{1}(t) & =2 b n_{M}(t)-k_{1} n_{1}(t), \\
\frac{d}{d t} n_{2}(t) & =n_{S}\left(t, T_{S}\right)-k_{2} n_{2}(t), \\
\frac{d}{d t} n_{M}(t) & =k_{2} n_{2}(t)-b n_{M}(t),
\end{aligned}
$$


for all $0<t<\infty$. Since $n_{S, 0}(\tau)$ is a continuously differentiable function on $\left[0, T_{S}\right]$ and

$$
n_{S}(t, \tau)= \begin{cases}n_{S, 0}(\tau-t) & \text { if } T_{S} \geq \tau \geq t \geq 0 \\ k_{1} n_{1}(t-\tau) & \text { if } \infty>t \geq \tau \geq 0\end{cases}
$$

We have

$$
\frac{\partial}{\partial t} n_{S}(t, \tau)+\frac{\partial}{\partial \tau} n_{S}(t, \tau)=0, \text { forall } 0<t<\infty, 0<\tau \leq T_{S}, t \neq \tau \text {. }
$$

We also have $n_{1}(0)=n_{1,0}, n_{2}(0)=n_{2,0}, n_{M}(0)=n_{M, 0}, n_{S}(0, \tau)=n_{S, 0}(\tau)$ for all $0 \leq \tau \leq T_{S}$ and $n_{S}(t, 0)=k_{1} n_{1}(t)$ for all $0 \leq t<\infty$. The assertion of this theorem is established. qed

Theorem 2.4. Suppose $n_{1,0}, n_{2,0}, n_{M, 0} \in \mathbb{R}$, and $n_{S, 0}(\tau)$ is a continuously differentiable function on $\left[0, T_{S}\right]$ with $n_{S, 0}(0)=k_{1} n_{1,0}$ and $\frac{d}{d \tau} n_{S, 0}(0)=k_{1}^{2} n_{1,0}-$ $2 b k_{1} n_{M, 0}$. Then the initial-boundary value problem (1.1)-(1.9) has a unique solution $\left(n_{1}(t), n_{S}(t, \tau), n_{2}(t), n_{M}(t)\right)$.

Proof. From Theorem 2.2 and Theorem 2.3, the system (2.1)-(2.4) has a unique solution $\left(n_{1}(t), n_{S}(t, \tau), n_{2}(t), n_{M}(t)\right)$. Moreover, $\left(n_{1}(t), n_{S}(t, \tau), n_{2}(t), n_{M}(t)\right)$ satisfies (1.1), (1.3)-(1.9) and $n_{S}(t, \tau)$ satisfy the differential equation (1.2) except possibly for $t=\tau$. Since $\frac{d}{d \tau} n_{S, 0}(0)=k_{1}^{2} n_{1,0}-2 b k_{1} n_{M, 0}$ and

$$
n_{S}(t, \tau)= \begin{cases}n_{S, 0}(\tau-t) & \text { if } T_{S} \geq \tau \geq t \geq 0, \\ k_{1} n_{1}(t-\tau) & \text { if } \infty>t \geq \tau \geq 0,\end{cases}
$$

both $\frac{\partial}{\partial t} n_{S}(t, \tau)$ and $\frac{\partial}{\partial \tau} n_{S}(t, \tau)$ exist, and they satisfy the equation $\frac{\partial}{\partial t} n_{S}(t, \tau)+$ $\frac{\partial}{\partial \tau} n_{S}(t, \tau)=0$ for all $0<t<\infty, 0<\tau \leq T_{S}$. The conclusion of this theorem follows.

\section{Growth Rate}

In this section we study the growth rate of the solution of the initial-boundary value problem (1.1)-(1.9). To achieve this goal, we need the following lemmas as preliminaries. In fact, they are crucial for this purpose. To simplify the notations, we denote by $C^{1}[0, \infty)$ and $C^{1}\left([0, \infty) \times\left[0, T_{S}\right]\right)$ as the sets of functions that are continuously differentiable in $[0, \infty)$ and $[0, \infty) \times\left[0, T_{S}\right]$, respectively.

Lemma 3.1. Suppose $w \in C^{1}\left([0, \infty) \times\left[0, T_{S}\right]\right)$ satisfies following inequalities

$$
\begin{cases}{[c] l \frac{\partial}{\partial \tau} w(t, \tau)+\frac{\partial}{\partial t} w(t, \tau) \geq 0} & \text { for all } 0<t<\infty, 0<\tau \leq T_{S} \\ w(0, \tau) \geq 0 & \text { for all } 0 \leq \tau \leq T_{S} \\ w(t, 0) \geq 0 & \text { for all } 0 \leq t<\infty .\end{cases}
$$


Then $w \geq 0$ on $[0, \infty) \times\left[0, T_{S}\right]$.

Proof. As $(t, \tau) \in[0, \infty) \times\left[0, T_{S}\right]$, we define $z(\eta)=w(t+\eta, \tau+\eta)$ for all $\max \{-t,-\tau\} \leq \eta \leq T_{S}-\tau$. Then $z^{\prime}(\eta)=w_{t}(t+\eta, \tau+\eta)+w_{\tau}(t+\eta, \tau+\eta) \geq$ 0 . If $t \geq \tau$, then $-\tau \leq \eta \leq 0 \leq T_{S}-\tau$ and hence $z(0)-z(-\tau)=\int_{-\tau}^{0} z^{\prime}(\eta) d \eta \geq$ 0 . This implies that $w(t, \tau) \geq w(t-\tau, 0) \geq 0$. On the other hand, if $t \leq \tau$, then $-t \leq \eta \leq 0 \leq T_{S}-\tau$ and hence $z(0)-z(-t)=\int_{-t}^{0} z^{\prime}(\eta) d \eta \geq 0$. These imply that $w(t, \tau) \geq w(0, \tau-t) \geq 0$. Therefore, $w \geq 0$ on $[0, \infty) \times\left[0, T_{S}\right]$, and the proof of this lemma is complete.

Lemma 3.2. Suppose $\gamma>0$ is a constant and $v \in C^{1}[0, \infty)$ satisfies following inequalities

$$
\left\{\begin{array}{l}
\frac{d}{d t} v(t)+\gamma v(t) \geq 0 \quad \text { for all } 0<t<\infty \\
v(0) \geq 0
\end{array}\right.
$$

Then $v \geq 0$ on $[0, \infty)$.

The proof of this lemma is trivial, we omit here.

We call a vector function

$$
\left(\widetilde{n}_{1}, \widetilde{n}_{S}, \widetilde{n}_{2}, \widetilde{n}_{M}\right) \in C^{1}[0, \infty) \times C^{1}\left([0, \infty) \times\left[0, T_{S}\right]\right) \times C^{1}[0, \infty) \times C^{1}[0, \infty)
$$

to be an upper solution of (1.1)-(1.9) if it satisfies the following inequalities:

$$
\begin{gathered}
\frac{d}{d t} \widetilde{n}_{1}(t)+k_{1} \widetilde{n}_{1}(t) \geq 2 b \widetilde{n}_{M}(t), \text { forall } 0<t<\infty, \\
\frac{\partial}{\partial \tau} \widetilde{n}_{S}(t, \tau)+\frac{\partial}{\partial t} \widetilde{n}_{S}(t, \tau) \geq 0, \text { forall } 0<t<\infty, 0<\tau \leq T_{S}, \\
\frac{d}{d t} \widetilde{n}_{2}(t)+k_{2} \widetilde{n}_{2}(t) \geq \widetilde{n}_{S}\left(t, T_{S}\right), \text { forall } 0<t<\infty, \\
\frac{d}{d t} \widetilde{n}_{M}(t)+b \widetilde{n}_{M}(t) \geq k_{2} \widetilde{n}_{2}(t), \text { forall } 0<t<\infty, \\
\widetilde{n}_{1}(0) \geq n_{1,0}, \\
\widetilde{n}_{S}(0, \tau) \geq n_{S, 0}(\tau), \text { forall } 0 \leq \tau \leq T_{S} \\
\widetilde{n}_{2}(0) \geq n_{2,0}, \\
\widetilde{n}_{M}(0) \geq n_{M, 0}, \\
\widetilde{n}_{S}(t, 0) \geq k_{1} \widetilde{n}_{1}(t), \text { forall } 0 \leq t<\infty .
\end{gathered}
$$


Similarly, $\left(\widehat{n}_{1}, \widehat{n}_{S}, \widehat{n}_{2}, \widehat{n}_{M}\right) \in C^{1}[0, \infty) \times C^{1}\left([0, \infty) \times\left[0, T_{S}\right]\right) \times C^{1}[0, \infty) \times$ $C^{1}[0, \infty)$ is called a lower solution if it satisfies the above inequalities in reversed order. The pair of upper solution and lower solution are said to be ordered if

$$
\left(\widetilde{n}_{1}, \widetilde{n}_{S}, \widetilde{n}_{2}, \widetilde{n}_{M}\right) \geq\left(\widehat{n}_{1}, \widehat{n}_{S}, \widehat{n}_{2}, \widehat{n}_{M}\right) .
$$

For a given pair of ordered upper and lower solutions, we set

$$
\begin{aligned}
\Omega & =\left\{\left(n_{1}, n_{S}, n_{2}, n_{M}\right) \in Y:\left(\widehat{n}_{1}, \widehat{n}_{S}, \widehat{n}_{2}, \widehat{n}_{M}\right)\right. \\
& \left.\leq\left(n_{1}, n_{S}, n_{2}, n_{M}\right) \leq\left(\widetilde{n}_{1}, \widetilde{n}_{S}, \widetilde{n}_{2}, \widetilde{n}_{M}\right)\right\}
\end{aligned}
$$

where $Y=C[0, \infty) \times C\left([0, \infty) \times\left[0, T_{S}\right]\right) \times C[0, \infty) \times C[0, \infty)$.

Given any initial iteration $\left(n_{1}^{(0)}, n_{S}^{(0)}, n_{2}^{(0)}, n_{M}^{(0)}\right)$ we can construct a sequence $\left(n_{1}^{(m)}, n_{S}^{(m)}, n_{2}^{(m)}, n_{M}^{(m)}\right), m \in \mathbb{N}$, according to the following iteration process:

$$
\begin{gathered}
\frac{d}{d t} n_{1}^{(m)}(t)+k_{1} n_{1}^{(m)}(t)=2 b n_{M}^{(m-1)}(t), \text { forall } 0<t<\infty \\
\frac{\partial}{\partial \tau} n_{S}^{(m)}(t, \tau)+\frac{\partial}{\partial t} n_{S}^{(m)}(t, \tau)=0, \text { forall } 0<t<\infty, 0<\tau \leq T_{S}, \\
\frac{d}{d t} n_{2}^{(m)}(t)+k_{2} n_{2}^{(m)}(t)=n_{S}^{(m-1)}\left(t, T_{S}\right), \text { forall } 0<t<\infty \\
\frac{d}{d t} n_{M}^{(m)}(t)+b n_{M}^{(m)}(t)=k_{2} n_{2}^{(m-1)}(t), \text { forall } 0<t<\infty \\
n_{1}^{(m)}(0)=n_{1,0}, \\
n_{S}^{(m)}(0, \tau)=n_{S, 0}(\tau), \text { forall } 0 \leq \tau \leq T_{S} \\
n_{2}^{(m)}(0)=n_{2,0}, \\
n_{M}^{(m)}(0)=n_{M, 0}, \\
n_{S}^{(m)}(t, 0)=k_{1} n_{1}^{(m-1)}(t), \text { forall } 0 \leq t<\infty .
\end{gathered}
$$

It is obviously that this sequence is well-defined and it can be represented as

$$
\begin{gathered}
n_{1}^{(m)}(t)=\exp \left(-k_{1} t\right) n_{1,0}+2 b \int_{0}^{t} \exp \left(-k_{1}(t-\eta)\right) n_{M}^{(m-1)}(\eta) d \eta, \\
n_{S}^{(m)}(t, \tau)=\left\{\begin{array}{l}
n_{S, 0}(\tau-t) \quad \text { if } \quad T_{S} \geq \tau \geq t \geq 0, \\
k_{1} n_{1}^{(m-1)}(t-\tau) \quad \text { if } \quad \infty>t \geq \tau \geq 0,
\end{array}\right.
\end{gathered}
$$




$$
n_{2}^{(m)}(t)=\exp \left(-k_{2} t\right) n_{2,0}+\int_{0}^{t} \exp \left(-k_{2}(t-\eta)\right) n_{S}^{(m-1)}\left(\eta, T_{S}\right) d \eta
$$

$$
n_{M}^{(m)}(t)=\exp (-b t) n_{M, 0}+k_{2} \int_{0}^{t} \exp (-b(t-\eta)) n_{2}^{(m-1)}(\eta) d \eta .
$$

We call $\left\{\left(\bar{n}_{1}^{(m)}, \bar{n}_{S}^{(m)}, \bar{n}_{2}^{(m)}, \bar{n}_{M}^{(m)}\right)\right\}$ a maximal sequence, if

$$
\left(n_{1}^{(0)}, n_{S}^{(0)}, n_{2}^{(0)}, n_{M}^{(0)}\right)=\left(\widetilde{n}_{1}, \widetilde{n}_{S}, \widetilde{n}_{2}, \widetilde{n}_{M}\right)
$$

and we call $\left\{\left(\underline{n}_{1}^{(m)}, \underline{n}_{S}^{(m)}, \underline{n}_{2}^{(m)}, \underline{n}_{M}^{(m)}\right)\right\}$ a minimal sequence, if

$$
\left(n_{1}^{(0)}, n_{S}^{(0)}, n_{2}^{(0)}, n_{M}^{(0)}\right)=\left(\widehat{n}_{1}, \widehat{n}_{S}, \widehat{n}_{2}, \widehat{n}_{M}\right) .
$$

The following lemma gives the monotone property of these sequences.

Lemma 3.3. The maximal and minimal sequences possess the following monotone properties:

$$
\begin{aligned}
& \widehat{n}_{1} \leq \underline{n}_{1}^{(m)} \leq \underline{n}_{1}^{(m+1)} \leq \bar{n}_{1}^{(m+1)} \leq \bar{n}_{1}^{(m)} \leq \widetilde{n}_{1}, \\
& \widehat{n}_{S} \leq \underline{n}_{S}^{(m)} \leq \underline{n}_{S}^{(m+1)} \leq \bar{n}_{S}^{(m+1)} \leq \bar{n}_{S}^{(m)} \leq \widetilde{n}_{S}, \\
& \widehat{n}_{2} \leq \underline{n}_{2}^{(m)} \leq \underline{n}_{2}^{(m+1)} \leq \bar{n}_{2}^{(m+1)} \leq \bar{n}_{2}^{(m)} \leq \widetilde{n}_{2}, \\
& \widehat{n}_{M} \leq \underline{n}_{M}^{(m)} \leq \underline{n}_{M}^{(m+1)} \leq \bar{n}_{M}^{(m+1)} \leq \bar{n}_{M}^{(m)} \leq \widetilde{n}_{M},
\end{aligned}
$$

for all $m \in \mathbb{N}$.

Proof. Let $w_{1}=\bar{n}_{1}^{(0)}-\bar{n}_{1}^{(1)}=\widetilde{n}_{1}-\bar{n}_{1}^{(1)}, w_{S}=\bar{n}_{S}^{(0)}-\bar{n}_{S}^{(1)}=\widetilde{n}_{S}-\bar{n}_{S}^{(1)}$, $w_{2}=\bar{n}_{2}^{(0)}-\bar{n}_{2}^{(1)}=\widetilde{n}_{2}-\bar{n}_{2}^{(1)}$ and $w_{M}=\bar{n}_{M}^{(0)}-\bar{n}_{M}^{(1)}=\widetilde{n}_{M}-\bar{n}_{M}^{(1)}$. Then

$$
\begin{aligned}
& \frac{d}{d t} w_{1}(t)+k_{1} w_{1}(t)=\left(\frac{d}{d t} \widetilde{n}_{1}(t)+k_{1} \widetilde{n}_{1}(t)\right)-2 b \widetilde{n}_{M}(t) \geq 0 \\
& \frac{d}{d t} w_{2}(t)+k_{2} w_{2}(t)=\left(\frac{d}{d t} \widetilde{n}_{2}(t)+k_{2} \widetilde{n}_{2}(t)\right)-\widetilde{n}_{S}\left(t, T_{S}\right) \geq 0 \\
& \frac{d}{d t} w_{M}(t)+b w_{M}(t)=\left(\frac{d}{d t} \widetilde{n}_{M}(t)+b \widetilde{n}_{M}(t)\right)-k_{2} \widetilde{n}_{2}(t) \geq 0 \\
& w_{1}(0)=\widetilde{n}_{1}(0)-n_{1,0} \geq 0, w_{2}(0)=\widetilde{n}_{2}(0)-n_{2,0} \geq 0, \\
& w_{M}(0)=\widetilde{n}_{M}(0)-n_{M, 0} \geq 0 .
\end{aligned}
$$


By Lemma 3.2, we have that $w_{1}(t) \geq 0, w_{2}(t) \geq 0$ and $w_{M}(t) \geq 0$ on $[0, \infty)$. Therefore,

$$
\begin{aligned}
\frac{\partial}{\partial t} w_{S}+\frac{\partial}{\partial \tau} w_{S} & =\left(\frac{\partial}{\partial t} \widetilde{n}_{S}+\frac{\partial}{\partial \tau} \widetilde{n}_{S}\right)-\left(\frac{\partial}{\partial t} \bar{n}_{S}^{(1)}+\frac{\partial}{\partial \tau} \bar{n}_{S}^{(1)}\right) \geq 0 \\
w_{S}(0, \tau) & =\widetilde{n}_{S}(0, \tau)-n_{S, 0}(\tau) \geq 0, \quad \text { for } 0 \leq \tau \leq T_{S} \\
w_{S}(t, 0) & =\widetilde{n}_{S}(t, 0)-\bar{n}_{S}^{(1)}(t, 0)=\widetilde{n}_{S}(t, 0)-k_{1} \bar{n}_{1}^{(0)}(t) \\
& \geq k_{1} \widetilde{n}_{1}(t)-k_{1} \widetilde{n}_{1}(t)=0,
\end{aligned}
$$

for all $0 \leq t<\infty$. By Lemma 3.1, $w_{S}(t, \tau) \geq 0$ on $[0, \infty) \times\left[0, T_{S}\right]$. This implies that

$$
\left(\bar{n}_{1}^{(1)}, \bar{n}_{S}^{(1)}, \bar{n}_{2}^{(1)}, \bar{n}_{M}^{(1)}\right) \leq\left(\bar{n}_{1}^{(0)}, \bar{n}_{S}^{(0)}, \bar{n}_{2}^{(0)}, \bar{n}_{M}^{(0)}\right) .
$$

A similar argument using the property of a lower solution gives

$$
\left(\underline{n}_{1}^{(0)}, \underline{n}_{S}^{(0)}, \underline{n}_{2}^{(0)}, \underline{n}_{M}^{(0)}\right) \leq\left(\underline{n}_{1}^{(1)}, \underline{n}_{S}^{(1)}, \underline{n}_{2}^{(1)}, \underline{n}_{M}^{(1)}\right) .
$$

Let $v_{1}^{(1)}=\bar{n}_{1}^{(1)}-\underline{n}_{1}^{(1)}, v_{S}^{(1)}=\bar{n}_{S}^{(1)}-\underline{n}_{S}^{(1)}, v_{2}^{(1)}=\bar{n}_{2}^{(1)}-\underline{n}_{2}^{(1)}$ and $v_{M}^{(1)}=\bar{n}_{M}^{(1)}-\underline{n}_{M}^{(1)}$.

\section{Then}

$$
\begin{aligned}
\frac{d}{d t} v_{1}^{(1)}+k_{1} v_{1}^{(1)} & =\left(\frac{d}{d t} \bar{n}_{1}^{(1)}+k_{1} \bar{n}_{1}^{(1)}\right)-\left(\frac{d}{d t} \underline{n}_{1}^{(1)}+k_{1} \underline{n}_{1}^{(1)}\right) \\
& =2 b\left(\widetilde{n}_{M}-\widehat{n}_{M}\right) \geq 0 \frac{d}{d t} v_{2}^{(1)}+k_{2} v_{2}^{(1)} \\
& =\left(\frac{d}{d t} \bar{n}_{2}^{(1)}+k_{2} \bar{n}_{2}^{(1)}\right)-\left(\frac{d}{d t} \underline{n}_{2}^{(1)}+k_{2} \underline{n}_{2}^{(1)}\right) \\
& =\widetilde{n}_{S}\left(t, T_{S}\right)-\widehat{n}_{S}\left(t, T_{S}\right) \geq 0 \\
\frac{d}{d t} v_{M}^{(1)}+b v_{M}^{(1)} & =\left(\frac{d}{d t} \bar{n}_{M}^{(1)}+b \bar{n}_{M}^{(1)}\right)-\left(\frac{d}{d t} \underline{n}_{M}^{(1)}+b \underline{n}_{M}^{(1)}\right)=k_{2}\left(\widetilde{n}_{2}-\widehat{n}_{2}\right) \geq 0 \\
v_{1}^{(1)}(0) & =n_{1,0}-n_{1,0}=0, v_{2}^{(1)}(0)=n_{2,0}-n_{2,0}=0, \\
v_{M}^{(1)}(0) & =n_{M, 0}-n_{M, 0}=0 .
\end{aligned}
$$

By Lemma 3.2, we have that $v_{1}^{(1)}(t) \geq 0, v_{2}^{(1)}(t) \geq 0$ and $v_{M}^{(1)}(t) \geq 0$ on $[0, \infty)$. Therefore, 


$$
\begin{aligned}
\frac{\partial}{\partial t} v_{S}^{(1)}+\frac{\partial}{\partial \tau} v_{S}^{(1)} & =\left(\frac{\partial}{\partial t} \bar{n}_{S}^{(1)}+\frac{\partial}{\partial \tau} \bar{n}_{S}^{(1)}\right)-\left(\frac{\partial}{\partial t} \underline{n}_{S}^{(1)}+\frac{\partial}{\partial \tau} \underline{n}_{S}^{(1)}\right) \geq 0 \\
v_{S}^{(1)}(0, \tau) & =n_{S, 0}(\tau)-n_{S, 0}(\tau)=0, \quad \text { for } 0 \leq \tau \leq T_{S} \\
v_{S}^{(1)}(t, 0) & =\bar{n}_{S}^{(1)}(t, 0)-\underline{n}_{S}^{(1)}(t, 0)=k_{1} \bar{n}_{1}^{(0)}(t)-k_{1} \underline{n}_{1}^{(0)}(t) \\
& =k_{1}\left(\widetilde{n}_{1}(t)-\widehat{n}_{1}(t)\right) \geq 0
\end{aligned}
$$

for $0 \leq t<\infty$. By Lemma 3.1, $v_{S}^{(1)}(t, \tau) \geq 0$ on $[0, \infty) \times\left[0, T_{S}\right]$. This implies that

$$
\left(\underline{n}_{1}^{(1)}, \underline{n}_{S}^{(1)}, \underline{n}_{2}^{(1)}, \underline{n}_{M}^{(1)}\right) \leq\left(\bar{n}_{1}^{(1)}, \bar{n}_{S}^{(1)}, \bar{n}_{2}^{(1)}, \bar{n}_{M}^{(1)}\right) \text {. }
$$

The above conclusions show that

$$
\begin{aligned}
& \left(\underline{n}_{1}^{(0)}, \underline{n}_{S}^{(0)}, \underline{n}_{2}^{(0)}, \underline{n}_{M}^{(0)}\right) \leq\left(\underline{n}_{1}^{(1)}, \underline{n}_{S}^{(1)}, \underline{n}_{2}^{(1)}, \underline{n}_{M}^{(1)}\right) \\
\leq & \left(\bar{n}_{1}^{(1)}, \bar{n}_{S}^{(1)}, \bar{n}_{2}^{(1)}, \bar{n}_{M}^{(1)}\right) \leq\left(\bar{n}_{1}^{(0)}, \bar{n}_{S}^{(0)}, \bar{n}_{2}^{(0)}, \bar{n}_{M}^{(0)}\right) .
\end{aligned}
$$

The monotone property (3.23)-(2.26) follows by an induction argument. The assertion of this lemma is proven.

In view of Lemma 3.3 the pointwise limits

$$
\begin{aligned}
& \lim _{m \rightarrow \infty}\left(\underline{n}_{1}^{(m)}, \underline{n}_{S}^{(m)}, \underline{n}_{2}^{(m)}, \underline{n}_{M}^{(m)}\right)=\left(\underline{n}_{1}, \underline{n}_{S}, \underline{n}_{2}, \underline{n}_{M}\right), \\
& \lim _{m \rightarrow \infty}\left(\bar{n}_{1}^{(m)}, \bar{n}_{S}^{(m)}, \bar{n}_{2}^{(m)}, \bar{n}_{M}^{(m)}\right)=\left(\bar{n}_{1}, \bar{n}_{S}, \bar{n}_{2}, \bar{n}_{M}\right)
\end{aligned}
$$

exist and satisfy

$$
\begin{aligned}
& \widehat{n}_{1} \leq \underline{n}_{1}^{(m)} \leq \underline{n}_{1}^{(m+1)} \leq \underline{n}_{1} \leq \bar{n}_{1} \leq \bar{n}_{1}^{(m+1)} \leq \bar{n}_{1}^{(m)} \leq \widetilde{n}_{1}, \\
& \widehat{n}_{S} \leq \underline{n}_{S}^{(m)} \leq \underline{n}_{S}^{(m+1)} \leq \underline{n}_{S} \leq \bar{n}_{S} \leq \bar{n}_{S}^{(m+1)} \leq \bar{n}_{S}^{(m)} \leq \widetilde{n}_{S}, \\
& \widehat{n}_{2} \leq \underline{n}_{2}^{(m)} \leq \underline{n}_{2}^{(m+1)} \leq \underline{n}_{2} \leq \bar{n}_{2} \leq \bar{n}_{2}^{(m+1)} \leq \bar{n}_{2}^{(m)} \leq \widetilde{n}_{2}, \\
& \widehat{n}_{M} \leq \underline{n}_{M}^{(m)} \leq \underline{n}_{M}^{(m+1)} \leq \underline{n}_{M} \leq \bar{n}_{M} \leq \bar{n}_{M}^{(m+1)} \leq \bar{n}_{M}^{(m)} \leq \widetilde{n}_{M},
\end{aligned}
$$

for every $m \in \mathbb{N}$. Letting $m \rightarrow \infty$ and using the integral representation of the sequences $\left\{\left(\bar{n}_{1}^{(m)}, \bar{n}_{S}^{(m)}, \bar{n}_{2}^{(m)}, \bar{n}_{M}^{(m)}\right)\right\},\left\{\left(\underline{n}_{1}^{(m)}, \underline{n}_{S}^{(m)}, \underline{n}_{2}^{(m)}, \underline{n}_{M}^{(m)}\right)\right\}$ show that both $\left(\bar{n}_{1}, \bar{n}_{S}, \bar{n}_{2}, \bar{n}_{M}\right)$ and $\left(\underline{n}_{1}, \underline{n}_{S}, \underline{n}_{2}, \underline{n}_{M}\right)$ are solutions the system (2.1)-(2.4). Since the system (2.1)-(2.4) has a unique solution $\left(n_{1}, n_{S}, n_{2}, n_{M}\right)$, it implies that

$$
\left(\bar{n}_{1}, \bar{n}_{S}, \bar{n}_{2}, \bar{n}_{M}\right)=\left(\underline{n}_{1}, \underline{n}_{S}, \underline{n}_{2}, \underline{n}_{M}\right)=\left(n_{1}, n_{S}, n_{2}, n_{M}\right) .
$$


Hence we have the following theorem.

Theorem 3.1 Let $\left(\widetilde{n}_{1}, \widetilde{n}_{S}, \widetilde{n}_{2}, \widetilde{n}_{M}\right)$ and $\left(\widehat{n}_{1}, \widehat{n}_{S}, \widehat{n}_{2}, \widehat{n}_{M}\right)$ be a pair of ordered upper and lower solutions of the differential system (1.1)-(1.9). Then the maximal and the minimal sequences $\left\{\left(\bar{n}_{1}^{(m)}, \bar{n}_{S}^{(m)}, \bar{n}_{2}^{(m)}, \bar{n}_{M}^{(m)}\right)\right\},\left\{\left(\underline{n}_{1}^{(m)}, \underline{n}_{S}^{(m)}, \underline{n}_{2}^{(m)}, \underline{n}_{M}^{(m)}\right)\right\}$ converge monotonically from above and below respectively to a unique solution $\left(n_{1}, n_{S}, n_{2}, n_{M}\right)$ of the system (2.1)-(2.4). Moreover,

$$
\begin{aligned}
& \widehat{n}_{1} \leq \underline{n}_{1}^{(m)} \leq \underline{n}_{1}^{(m+1)} \leq n_{1} \leq \bar{n}_{1}^{(m+1)} \leq \bar{n}_{1}^{(m)} \leq \widetilde{n}_{1}, \\
& \widehat{n}_{S} \leq \underline{n}_{S}^{(m)} \leq \underline{n}_{S}^{(m+1)} \leq n_{S} \leq \bar{n}_{S}^{(m+1)} \leq \bar{n}_{S}^{(m)} \leq \widetilde{n}_{S}, \\
& \widehat{n}_{2} \leq \underline{n}_{2}^{(m)} \leq \underline{n}_{2}^{(m+1)} \leq n_{2} \leq \bar{n}_{2}^{(m+1)} \leq \bar{n}_{2}^{(m)} \leq \widetilde{n}_{2}, \\
& \widehat{n}_{M} \leq \underline{n}_{M}^{(m)} \leq \underline{n}_{M}^{(m+1)} \leq n_{M} \leq \bar{n}_{M}^{(m+1)} \leq \bar{n}_{M}^{(m)} \leq \widetilde{n}_{M},
\end{aligned}
$$

for all $m \in \mathbb{N}$.

Based on Theorem 3.1 and a suitable construction of a lower solution we can obtain a lower bound of the solution for the initial-boundary value problem (1.1)(1.9).

Theorem 3.2. Suppose that $n_{1,0}>0, n_{2,0}>0, n_{M, 0}>0, \min _{\tau \in\left[0, T_{S}\right]} n_{S, 0}(\tau)>0$. Then the solution $\left(n_{1}(t), n_{S}(t, \tau), n_{2}(t), n_{M}(t)\right)$ of the initial-boundary value problem (1.1)-(1.9) with the initial condition $\left(n_{1,0}, n_{S, 0}(\tau), n_{2,0}, n_{M, 0}\right)$ satisfies that

$n_{1}(t) \geq \frac{\rho}{k_{1}} e^{\varepsilon t}, n_{S}(t, \tau) \geq \rho e^{\varepsilon(t-\tau)}, n_{2}(t) \geq \frac{(1+\delta)^{2}}{2 k_{2}} \rho e^{\varepsilon t}$ and $n_{M}(t) \geq \frac{1+\delta}{2 b} \rho e^{\varepsilon t}$

for all $t \in[0, \infty)$ and $\tau \in\left[0, T_{S}\right]$, where $0<\varepsilon \leq \min \left\{\delta k_{1}, \delta k_{2}, \delta b, \frac{1}{T_{S}} \ln \frac{2}{(1+\delta)^{3}}\right\}$, $0<\delta<\sqrt[3]{2}-1$ and $0<\rho \leq \min \left\{k_{1} n_{1,0}, \min _{\tau \in\left[0, T_{S}\right]} n_{S, 0}(\tau), \frac{2 k_{2} n_{2,0}}{(1+\delta)^{2}}, \frac{2 b n_{M, 0}}{1+\delta}\right\}$.

Proof. Let $\widehat{n}_{1}(t)=\frac{\rho}{k_{1}} e^{\varepsilon t}, \widehat{n}_{S}(t, \tau)=\rho e^{\varepsilon(t-\tau)}, \widehat{n}_{2}(t)=\frac{(1+\delta)^{2}}{2 k_{2}} \rho e^{\varepsilon t}$ and $\widehat{n}_{M}(t)=\frac{1+\delta}{2 b} \rho e^{\varepsilon t}$. Then

$$
\begin{aligned}
\widehat{n}_{1}(0) & =\frac{\rho}{k_{1}} \leq n_{1,0}, \widehat{n}_{2}(0)=\frac{(1+\delta)^{2}}{2 k_{2}} \rho \leq n_{2,0}, \widehat{n}_{M}(0)=\frac{1+\delta}{2 b} \rho \leq n_{M, 0}, \\
\widehat{n}_{S}(0, \tau) & =\rho e^{-\varepsilon \tau} \leq \rho \leq \min _{\tau \in\left[0, T_{S}\right]} n_{S, 0}(\tau) \leq n_{S, 0}(\tau) \text { and } \\
\widehat{n}_{S}(t, 0) & =\rho e^{\varepsilon t}=k_{1} \widehat{n}_{1}(t) .
\end{aligned}
$$


According to the assumptions and the definition of the functions $\widehat{n}_{1}(t), \widehat{n}_{S}(t, \tau)$, $\widehat{n}_{2}(t), \widehat{n}_{M}(t)$, we have

$$
\begin{aligned}
& \frac{d}{d t} \widehat{n}_{1}(t)+k_{1} \widehat{n}_{1}(t)-2 b \widehat{n}_{M}(t)=\varepsilon \frac{\rho}{k_{1}} e^{\varepsilon t}+\rho e^{\varepsilon t}-(1+\delta) \rho e^{\varepsilon t} \\
& =\frac{\rho}{k_{1}}\left[\varepsilon+k_{1}-(1+\delta) k_{1}\right] e^{\varepsilon t} \\
& =\frac{\rho}{k_{1}}\left[\varepsilon-\delta k_{1}\right] e^{\varepsilon t} \\
& \leq 0 \\
& \frac{\partial}{\partial \tau} \widehat{n}_{S}(t, \tau)+\frac{\partial}{\partial t} \widehat{n}_{S}(t, \tau)=-\varepsilon \rho e^{\varepsilon(t-\tau)}+\varepsilon \rho e^{\varepsilon(t-\tau)}=0, \\
& \frac{d}{d t} \widehat{n}_{2}(t)+k_{2} \widehat{n}_{2}(t)-\widehat{n}_{S}\left(t, T_{S}\right)=\frac{(1+\delta)^{2}}{2 k_{2}} \varepsilon \rho e^{\varepsilon t}+\frac{(1+\delta)^{2}}{2} \rho e^{\varepsilon t}-\rho e^{-\varepsilon T_{S}} e^{\varepsilon t} \\
& =\frac{(1+\delta)^{2}}{2 k_{2}} \rho\left[\varepsilon+k_{2}-\frac{2 k_{2}}{(1+\delta)^{2}} e^{-\varepsilon T_{S}}\right] e^{\varepsilon t} \\
& \leq \frac{(1+\delta)^{2}}{2 k_{2}} \rho\left[\varepsilon+k_{2}-\frac{k_{2}}{(1+\delta)^{2}}(1+\delta)^{3}\right] e^{\varepsilon t} \\
& =\frac{(1+\delta)^{2}}{2 k_{2}} \rho\left[\varepsilon-\delta k_{2}\right] e^{\varepsilon t} \\
& \leq 0 \\
& \frac{d}{d t} \widehat{n}_{M}(t)+b \widehat{n}_{M}(t)-k_{2} \widehat{n}_{2}(t)=\varepsilon \frac{1+\delta}{2 b} \rho e^{\varepsilon t}+\frac{1+\delta}{2} \rho e^{\varepsilon t}-\frac{(1+\delta)^{2}}{2} \rho e^{\varepsilon t} \\
& =\frac{1+\delta}{2 b} \rho[\varepsilon+b-(1+\delta) b] e^{\varepsilon t} \\
& =\frac{1+\delta}{2 b} \rho[\varepsilon-\delta b] e^{\varepsilon t} \\
& \leq 0 .
\end{aligned}
$$

This implies that $\left(\widehat{n}_{1}, \widehat{n}_{S}, \widehat{n}_{2}, \widehat{n}_{M}\right)$ is a lower solution of the system (1.1)-(1.4). Hence

$$
\begin{aligned}
& n_{1}(t) \geq \frac{\rho}{k_{1}} e^{\varepsilon t}, n_{S}(t, \tau) \geq \rho e^{\varepsilon(t-\tau)}, n_{2}(t) \geq \frac{(1+\delta)^{2}}{2 k_{2}} \rho e^{\varepsilon t} \text { and } n_{M}(t) \geq \frac{1+\delta}{2 b} \rho e^{\varepsilon t} \\
& \text { for all } t \in[0, \infty) \text { and } \tau \in\left[0, T_{S}\right] . \text { The proof of this theorem is completed. }
\end{aligned}
$$

\section{Conclusion And Discussion}

From the closed form of unperturbed cell line population functions

$$
n_{1}(t)=\exp \left(-k_{1} t\right) n_{1,0}+2 b \int_{0}^{t} \exp \left(-k_{1}(t-\eta)\right) n_{M}(\eta) d \eta,
$$




$$
\begin{gathered}
n_{S}(t, \tau)=\left\{\begin{array}{cc}
n_{S, 0}(\tau-t) & \text { if } T_{S} \geq \tau \geq t \geq 0, \\
k_{1} n_{1}(t-\tau) & \text { if } \infty>t \geq \tau \geq 0,
\end{array}\right. \\
n_{2}(t)=\exp \left(-k_{2} t\right) n_{2,0}+\int_{0}^{t} \exp \left(-k_{2}(t-\eta)\right) n_{S}\left(\eta, T_{S}\right) d \eta, \\
n_{M}(t)=\exp (-b t) n_{M, 0}+k_{2} \int_{0}^{t} \exp (-b(t-\eta)) n_{2}(\eta) d \eta,
\end{gathered}
$$

the populations in each phase depend on the initial functions and the transition coefficients. The work explains why the populations in each phase of different unperturbed cell lines are distributed differently. Furthermore, according to (2.1)(2.4), if the populations of human tumor cells in each of $G_{1^{-}}, S-, G_{2^{-}}$and $M$-phase of unperturbed cells at a certain time can be understood, it is then predictable how those in each phase of unperturbed cells be like.

On the other hand, the results can be further extended to compare with the phase distributions in tumor cells under anticancer treatments and hence to predict their efficiency. For instance, in view of theorem 3.2 the growth rate of the populations in each phase of unperturbed human tumor cells is faster than exponential functions which depend on the initial functions, the transition coefficients and the time of the cells in the $S$-phase respectively. Specifically, we have

$$
\begin{aligned}
n_{1}(t) & \geq \frac{\rho}{k_{1}} e^{\varepsilon t}, n_{S}(t, \tau) \geq \rho e^{\varepsilon(t-\tau)}, n_{2}(t) \\
& \geq \frac{(1+\delta)^{2}}{2 k_{2}} \rho e^{\varepsilon t} \text { and } n_{M}(t) \geq \frac{1+\delta}{2 b} \rho e^{\varepsilon t}
\end{aligned}
$$

for all $t \in[0, \infty)$ and $\tau \in\left[0, T_{S}\right]$, where $0<\varepsilon \leq \min \left\{\delta k_{1}, \delta k_{2}, \delta b, \frac{1}{T_{S}} \ln \frac{2}{(1+\delta)^{3}}\right\}$, $0<\delta<\sqrt[3]{2}-1$ and $0<\rho \leq \min \left\{k_{1} n_{1,0}, \min _{\tau \in\left[0, T_{S}\right]} n_{S, 0}(\tau), \frac{2 k_{2} n_{2,0}}{(1+\delta)^{2}}, \frac{2 b n_{M, 0}}{1+\delta}\right\}$. However, in [7] we showed that, under the treatment by radiotherapy or chemotherapy, if one can suitably control the losing rates $\mu_{1}$ and $\mu_{M}$ of the cells in the $G_{1}$-phase and in the $M$-phase respectively, or one may control the time of the cells in the $S$-phase, then the inequality

$$
k_{1} k_{2} k_{M} e^{-\mu_{S} T_{S}}\left[\frac{3}{2}+\frac{\sqrt{\alpha}}{\beta \sqrt{T_{S} \pi}}\left(1+O\left(T_{S}^{-1}\right)\right)\right]<\mu_{1}\left(\mu_{1}-\mu_{2}\right)\left(\mu_{M}-\mu_{2}\right)
$$

hold. The physical meanings of the positive constants $k_{M}, \mu_{1}, \mu_{2}, \mu_{S}, \mu_{M}, T_{S}$, $\alpha$ and $\beta$ are described in the introduction section of [7]. In there, we show that if $\mu_{1}>\mu_{2}, \mu_{M}>\mu_{2}$, and the inequality (4.2) hold, then the DNA of tumour cells $G_{1}(x, t)$, and hence the population of tumour cells $n_{1}(t)=\int_{0}^{L} G_{1}(x, t) d x$, in $G_{1}$-phase decays exponentially with time $t$ for arbitrarily fixed $x \in[0, \infty)$ (please 
see [7, Theorem 6], for detail). Thus the growth of human tumour cells will be controlled under therapy. On the other hand, in [7] we also got

$$
\begin{aligned}
& G_{1}(x, t)=e^{-\mu_{1} t} G_{1}(x, 0) \\
& \quad+k_{M} k_{2}\left[\int_{0}^{t} \int_{\xi}^{t} \int_{\eta}^{t} e^{\mu_{1}(\tau-t)} e^{\mu_{M}(\eta-\tau)} e^{\mu_{2}(\xi-\eta)} I\left(2 x, \xi, T_{S}\right) d \tau d \eta d \xi\right]
\end{aligned}
$$

This shows that the DNA of tumour cells $G_{1}(x, t)$ in $G_{1}$-phase are depending on the initial function $G_{1}(x, 0)$ under the treatment by radiotherapy or chemotherapy. Inequality (4.1) shows that the growth rate of the population in each phase of unperturbed human tumour cells is faster than exponential functions, while the inequalities (4.2) and (4.3) indicate that the population in each phase of perturbed human tumour cells decays exponentially with time $t$ for arbitrarily fixed $x \in[0, \infty)$. Since all the inequalities (4.1)-(4.3) depend on their initial data, these facts show that early therapy should be a better choice in general.

\section{ACKNOWLEDGMENTS}

The authors would like to express their gratitude to the reviewers for the very useful suggestions. We also like to express our gratitude to professor C. V. Pao for his useful suggestions and communications.

\section{REFERENCES}

1. B. Basse, B. C. Baguley, E. S. Marshall, W. R. Joseph, B. van Brunt, G. C. Wake and D. J. N. Wall, A mathematical model for analysis of the cell cycle in cell lines derived from human tumors, J. Math. Biol., 47 (2003), 295-312.

2. B. Basse, B. C. Baguley, E. S. Marshall, W. R. Joseph, B. van Brunt, G. C. Wake and D. J. N. Wall, Modelling cell death in human tumour cell lines exposed to the anticancer drug paclitaxel, J. Math. Biol., 49 (2004a), 329-357.

3. B. Basse, B. C. Baguley, E. Marshall, G. C. Wake and D. J. N. Wall, Modelling cell population growth with applications to cancer therapy in human tumour cell lines, Prog. Biophys. Mol. Biol., 85 (2004b), 353-368.

4. B. Basse, G. C. Wake, D. J. N. Wall and B. van Brunt, On a cell growth model for plankton, Math. Med. Biol. J. IMA, 21 (2004c), 49-61.

5. B. Basse, B. C. Baguley, E. S. Marshall, G. C. Wake and D. J. N. Wall, Modelling the flow of cytometric data obtained from unperturbed human tumor cell lines: parameter fitting and comparison, Bulletin of Math. Biol., 67 (2005), 815-830.

6. G. Bell and E. Anderson, Cell growth and division. Mathematical model with applications to cell volume distributions in mammalian suspension cultures, Biophys. J., 7 (1967), 329-351. 
7. Y. H. Chang and G. C. Jau, The behavior of the solution to a mathematical model for analysis of the cell cycle, CAPP, Vol. 5, No. 4, December, 2006, pp. 779-792.

8. G. Chiorino, J. A. J. Metz, D. Tomasoni and P. Ubezio, Desynchronization rate in cell populations: mathematical modeling and experimental data, J. Theor. Biol., 208 (2001), 185-199.

9. G. Chiorini and M. Lupi, Variability in the timing of G1/S transition, Math. Biosci., (2002), in press.

10. O. Diekmann, Growth, fission and the stable size distribution, J. Math. Biol., 18 (1983), 135-148.

11. O. Diekmann, H. J. A. M. Heijmans and H. R. Thieme, On the stability of the cell size distribution, J. Math. Biol., 19 (1984), 227-248.

12. M. Gyllenberg and G. F. Webb, A nonlinear structured population model of tumor growth with quiescence, J. Math. Biol., 28 (1990), 671-694.

13. K. B. Hannsgen and J. J. Tyson, Stability of the steady-state size distribution in a model of cell growth and division, J. Math. Biol., 22 (1985), 293-301.

14. E. Kreyszig, Introductory Functional Analysis with Applications, Springer-Verlag, New York, 1978.

15. R. H. Martin Jr., Nonlinear Operators and Differential Equations in Banach Spaces, Academic Press, New York, 1976.

16. C. McCluer, The many proofs and applications of Perron's theorem, SIAM Rev., 42(3) (2000), 487-498.

17. F. Montalenti, G. Sena, P. Cappella and P. Ubezio, Simulating cancer-cell kinetics after drug treatment: Application to cisplatin on ovarian carcinoma, Phys. Rev. E, 57(5) (1999), 5877-5887.

18. C. V. Pao, Nonlinear Parabolic and Elliptic Equations, Plenum Press, New York, 1992.

19. A. Pazy, Semigroup of Linear Operators and Applications to Partial Differential Equations, Springer-Verlag, New York, 1983.

20. L. Priori and P. Ubezio, Mathematical modelling and computer simulation of cell synchrony, Methods in cell science, 18 (1996), 83-91.

21. B. Rossa, Asynchronous exponential growth in a size structured cell population with quiescent compartment, Carcinogenesis and Cell \& Tumor Growth of Arino et al. 1995, Vol. 2. (Chapter 14), 1995, pp. 183-200.

22. G. Sena, C. Onado, P. Cappella, F. Montalenti and P. Ubezio, Measuring the complexity of cell cycle arrest and killing of drugs: Kinetics of phase-specific effects induced by taxol, Cytometry, 37 (1999), 113-124.

23. S. Sinko and W. Streifer, A new model for age-size structure of a population, Ecology, 48 (1967), 330-335. 
24. M. Takahashi, Theoretical basis for cell cycle analysis. II. Further studies on labelled mitosis wave method, J. Theor. Biol., 18 (1968), 195-209.

25. P. Ubezio, Cell cycle simulation for flow cytometry. Computer methods and programs in biomedicine. Section II, Systems and programs, 31(3697) (1990), 255-266.

26. P. Ubezio, Relationship between flow cytometric data and kinetic parameters, Eur. J. Histochem., 37(Suppl. 4) (1993), 15-28.

27. P. Ubezio, Unraveling the complexity of cell cycle effects of anticancer drugs in cell populations, Discrete Contin. Dyn. Sys. Ser., B4(1) (2004), 323-335.

\section{Yu-Hsien Chang}

Department of Mathematics

National Taiwan Normal University

Taipei 116, Taiwan

E-mail: changyh@math.ntnu.edu.tw

\section{Kang Fang}

Department of Life Science

National Taiwan Normal University

Taipei 116, Taiwan

E-mail: kangfang@math.ntnu.edu.tw

\section{Guo-Chin Jau}

Department of Mathematics and Computer Science Education

Taipei Municipal University of Education

Taipei 100, Taiwan

E-mail: jaugc@tmue.edu.tw 